\title{
DESCRIPCIÓN DE ANORMALIDAD DE LA CITOLOGÍA CÉRVICO UTERINA EN ARMENIA - QUINDÍO EN EL AÑO 2012
}

\author{
DESCRIPTION OF ABNORMAL UTERINE CERVICAL \\ CITOLOGY IN ARMENIA - QUINDÍO IN 2012
}

Manuel Fernando Hincapié Aranzazu ${ }^{1,2}$, Angélica María López Montoya ${ }^{1,2}$, Kelly Johanna Castaño Lezcano ${ }^{1,2}$, Olga Alicia Nieto Cárdenas ${ }^{1,2}$.

1. Programa de Medicina. Facultad de Ciencias de la Salud. Universidad del Quindío.

2. Grupo de investigación en Salud Pública. Facultad de Ciencias de la Salud. Universidad del Quindío.

Recibido: 5 de Septiembre de 2014 Aceptado: 25 de Septiembre de 2014

*Correspondencia del autor: Universidad del Quindío, Avenida Bolívar calle 12 norte Armenia Quindío Colombia. Email: manuel.hinkpie@gmail.com

\section{RESUMEN}

A pesar que existe una prueba tamiz, que permite detectar a tiempo el cáncer de cuello uterino, muchas mujeres no la realizan. Específicamente en mujeres de Armenia no se conocen estudios que muestren la situación actual de realización de la citología cérvico uterina.

Objetivo: describir los resultados obtenidos en reportes de citología realizados en el año 2012 y aportar a la construcción de la línea base del Plan Decenal de Salud para Armenia.

Métodos: estudio descriptivo de corte transversal, realizado con la Secretaria de Salud, quien aportó la información y el aplicativo para su sistematización. Se tomó una muestra aleatoria del total de los reportes de citología, se sistematizó y analizó la información.

Resultados: Se encontró que las mujeres tenían edades entre 15 y 79 años, en promedio 37,4; el resultado más reportado fue Atipia celular (ASC-US) (76\%), el manejo que más se recomendó fue biopsia más colposcopia (36,05\%); el 22,37\% no utilizaba ningún método de planificación familiar; la institución que más realizó citologías fue Quindicáncer $(20,79 \%)$ y el resultado atipia celular reportó con mayor frecuencia el manejo biopsia más colposcopia (25,79\%). El 16,05\% de las mujeres con este resultado no utilizaban ningún método de planificación y sólo el 1,52 \% utilizaba condón.

Conclusión: las mujeres de Armenia presentaron en mayor porcentaje el resultado atipia celular y se les recomendó el manejo biopsia más colposcopia, indicado según literatura, no utilizaban métodos de planificación familiar y sólo el 1,52\% que se hizo la citología utilizaba protección con condón.

Palabras claves: citología cérvico uterina, anormalidad en citología 


\begin{abstract}
Although there is a screening test that allows early detection of cervical cancer, many women do not do it. Specifically in women of Armenia, there are no studies showing the current status of implementation of cervical cytology.

Objective: To describe the results of cytology reports made in 2012 and contribute to the construction of the base line of the Ten-Year Health Plan for Armenia.

Methods: A descriptive cross-sectional study, conducted hand in hand with the Secretary of Health, which provided information and application for systematization. A random sample of all cytology reports were taken, and the information was analyzed and systematized-

Results: We found that women were aged between 15 and 79 years; the average was 37.4 years old. The most reported result was cellular atypia (ASC-US) (76\%). The most commonly recommended treatment was colposcopy + biopsy $(36.05 \%) ; 22.37 \%$ of those women were not using any contraceptive methods. Quindicancer was the institution that performed most of the cytology $(20.79 \%)$ and cellular atypia resulted frequently reported the treatment with colposcopy + biopsy $(25.79 \%)$. The $16.05 \%$ of women with this result did not use any contraceptive method and only $1.52 \%$ used condom.

Conclusion: Women in Armenia presented a higher percentage of cellular atypia and colposcopy + biopsy were advised as indicated in literature, most of the did not use contraceptive methods and only $1.52 \%$ who did the cytology used condom
\end{abstract}

Keywords: uterine cervical cytology, abnormality in cytology.

\section{INTRODUCCIÓN}

La presente investigación es sobre los resultados de la citología cérvicouterina la cual es una prueba tamiz para la detección temprana del cáncer de cuello uterino dirigida a las mujeres entre 25 y 69 años o menores de 25 años con vida sexual activa. La citología cérvicouterina es de vital importancia ya que el cáncer de cuello uterino es un grave problema de salud pública porque presenta altas cifras de incidencia; estadísticamente es el segundo cáncer más frecuente en el mundo con 500.000 casos nuevos en el año y 274.000 muertes anuales, precedido por el cáncer de mama en la población femenina. En Colombia, según estadísticas del Instituto Nacional de Cancerología (INC), se reporta que esta enfermedad constituye aproximadamente el $16 \%$ de los casos nuevos, lo que lo convierte en la primera causa de incidencia y mortalidad entre nuestras mujeres (1). La detección precoz del cáncer de cuello uterino genera una notable reducción de la mortalidad y permite intervenciones tempranas ante procesos de posible malignidad.

Para el desarrollo de este proyecto, la Secretaria de Salud Municipal de Armenia aportó los reportes de las citologías cérvico uterinas realizadas, de todas las EPS e IPS de Armenia Quindío, durante el año 2012 y el aplicativo para su sistematización. Se sistematizó la información del total de las 841 citologías que habían sido reportadas con algún grado de anormalidad y se tomó una muestra de 384 mujeres entre los 15 a 79 años de edad pertenecientes al municipio de Armenia Quindío que se han realizado la citología cérvico uterina en el año 2012. Con estos datos se pudo tener acceso a una información actualizada para así determinar con respecto a la citología cérvico uterina en qué contexto se encuentra nuestro municipio y poder generar ideas de intervención que disminuyan la incidencia del cáncer de cuello uterino.

La presente investigación describe los resultados obtenidos en los reportes de citologías realizados en la ciudad de Armenia en el año 2012 y pretende aportar a la construcción de la línea base del Plan Decenal de Salud para la Ciudad de Armenia.

\section{MATERIALES Y MÉTODOS Área y tipo de estudio}

Se realizó un estudio descriptivo de corte transversal, en el municipio de Armenia Quindío - Colombia.

\section{Población y sujetos de estudio}

Se incluyeron en el estudio una población de $841 \mathrm{mu}-$ jeres (entre 15 y 79 años) que se realizaron la citología cérvico uterina en las diferentes EPS e IPS del municipio de Armenia durante el año 2012, cuyos re- 
sultados reportaron algún grado de anormalidad y se calculó una muestra aleatoria de 384 mujeres.

\section{Recolección de la información}

El proyecto tuvo dos momentos, uno en el cual se presentó para aprobación institucional y el otro la recolección de la información. Se presentó el protocolo de investigación como parte de la asignatura de Investigación de VIII y IX semestre de Medicina en la Universidad del Quindío, para el acompañamiento bioético y el consentimiento institucional por el director del programa. Posteriormente se presentó el protocolo de investigación a la Secretaría de Salud del municipio de Armenia ante la Dirección de Salud Pública.

Una vez fue aprobado el proyecto, la Secretaría de Salud municipal aportó los reportes de la citología cérvico uterina de todas las EPS de Armenia Quindío del año 2012. La información fue recolectada por los investigadores en el aplicativo en Excel diseñado por la Secretaría de Salud la Alcaldía del municipio de Armenia y fue procesada en el primer semestre del 2014.

\section{Procesamiento de la información}

Se sistematizó la información en el aplicativo en Excel y posteriormente se procesó, analizó y se realizaron las gráficas por medio del software Statgraphics Centurión 15. Las variables numéricas se presentan en promedio, desviación estándare Intervalo de confianza (IC95\%); se organizaron en tablas distribución de frecuencia para datos agrupados y se hicieron gráficos de histogramas y cajas y bigotes. Las variables categóricas, se presentan en moda y porcentaje. Se hizo una prueba de chi cuadrado para ver la relación entre los resultados de las citologías con porcentaje de anormalidad, manejo, método de planificación y EPS e IPS.

\section{RESULTADOS}

Se incluyeron 841 reportes de citologías anormales, enviadas a la Secretaría de Salud de Armenia por las diferentes EPS e IPS de la ciudad, del total de 61.037 citologías realizadas en el 2012 (1,37\% de anormalidad).

Sin embargo, si calculamos sobre los grupos de edad de las mujeres del presente estudio (15-75 años), el porcentaje de anormalidad es de $0,75 \%$.

Es decir, el porcentaje de anormalidad de citologías en la información acopiada por la Secretaría de Salud entre las diferentes EPS e IPS de la ciudad es menor del $1 \%$, de la población a riesgo.

Se calculó una muestra para una población infinita, por cuanto se hizo para el total de la población de mujeres que estaban en edad de hacerse la citología, es decir 384 reportes, que fueron seleccionados mediante una tabla de números aleatorios en la base de datos de la Secretaría de Salud (2), cuya información se procesó y los resultados se describen a continuación; en la tabla lencontramos las variables numéricas de los reportes de citología.

Tabla 1. Variables numéricas de los reportes de citología Armenia 2012

\begin{tabular}{lccc}
\hline Variable & Promedio (X) & $\begin{array}{c}\text { Desviación } \\
\text { estándar (S) }\end{array}$ & IC95\% \\
\hline EDAD & 37,44 & 13,09 & $36,11-38,77$ \\
GESTA- & 1,86 & 1,35 & $1,66-2,06$ \\
CION & & & \\
PARIDAD & 1,74 & 1,29 & $1,53-1,93$ \\
\hline
\end{tabular}

Se puede observar que las mujeres participantes del estudio tienen una edad en promedio de 37,4 años, con un mínimo de 15 y un máximo de 79 años de edad. Encontramos que el rango de edad entre 20 años y 30 años es el valor que más se repite con 110 datos que corresponden en total a un $29 \%$ y una media de 25 años. Seguidas por el rango de 40-50 años, con una media de 45 años y 89 datos que corresponden al 24\%, 30-40 años con una media de 35 años y 87 datos $(23 \%)$ y por último el rango de 50-60 años con una media de 55 años y 45 datos que corresponden al $12 \%$.

El promedio de gestaciones fue de 1,86 con un mínimo de 0 y un máximo de 6 gestaciones. El número de gestaciones que más se repitió equivale a tres gestaciones en 52 mujeres (30,5\%), en segundo lugar dos gestaciones en 43 mujeres $(25,2 \%)$, le sigue una única gestación en $28(16,47 \%)$ pacientes, posteriormente 27 mujeres con cuatro gestaciones $(15,88 \%)$, continua cinco gestaciones en $13(0,72 \%)$ mujeres y por último siete y ocho gestaciones en $5(0,29)$ y 2 $(0,11 \%)$ respectivamente. El promedio de partos fue de 1,73 con un mínimo de 0 y un máximo de 6 partos y el dato de partos que más se repite equivale a tres partos en 59 mujeres $(34,7 \%)$, en segundo lugar dos partos en 47 mujeres $(27.6 \%)$, le sigue un único parto en 28 mujeres $(17,06 \%)$, posteriormente 19 mujeres con cuatro partos $(11,1 \%)$, continua cinco partos en 9 
Tabla 2. Variables categóricas de los reportes de citología de Armenia 2012

\begin{tabular}{|c|c|c|c|c|}
\hline Variable & Moda & $\begin{array}{c}\text { No } \\
\text { Categorías }\end{array}$ & Categorías & Porcentaje \\
\hline RESULTADOS DE CITOLOGIA & $\begin{array}{c}\text { Atipias celulares } \\
289\end{array}$ & 8 & $\begin{array}{c}\text { Atipias celulares } \\
\text { LEIBG } \\
\text { LEIAG } \\
\text { Infección por VPH } \\
\text { Neoplasia } \\
\text { intraepitelial } \\
\text { Otras neoplasias } \\
\text { Cambios celulares } \\
\text { Negativa para neoplasia }\end{array}$ & $\begin{array}{c}76 \% \\
16 \% \\
5 \% \\
0,08 \% \\
0,05 \% \\
\\
0,05 \% \\
0,03 \%\end{array}$ \\
\hline MANEJO & $\begin{array}{c}\text { Biopsia }+ \\
\text { colposcopia } \\
137\end{array}$ & 5 & $\begin{array}{c}\text { Biopsia }+ \text { colposcopia } \\
\text { NR } \\
\text { Remisión a Ginecología } \\
\text { Colposcopia } \\
\text { Biopsia }\end{array}$ & $\begin{array}{c}36,05 \% \\
29,47 \% \\
18,16 \% \\
15,79 \% \\
0,05 \%\end{array}$ \\
\hline METODOS PLANIFICACION & NR 134 & 8 & $\begin{array}{c}\text { NR } \\
\text { Ninguno } \\
\text { Quirúrgico } \\
\text { Hormonal inyectable } \\
\text { Hormonal oral } \\
\text { DIU } \\
\text { De barrera } \\
\text { Hormonal implante }\end{array}$ & $\begin{array}{c}35,26 \% \\
22,37 \% \\
18,42 \% \\
8,95 \% \\
8,42 \% \\
3,16 \% \\
2,11 \% \\
1,32 \%\end{array}$ \\
\hline EPS & NR 112 & 15 & $\begin{array}{l}\text { NR } \\
\text { Quindicancer } \\
\text { Sanitas S.A. } \\
\text { Cosmitet } \\
\text { Coomeva EPS } \\
\text { Saludcoop EPS } \\
\text { Comfenalco Antioquia } \\
\text { Cafesalud EPS } \\
\text { Red salud } \\
\text { Salud total } \\
\text { Comfenalco Quindío } \\
\text { Uniquindío } \\
\text { Caprecom } \\
\text { Sanidad militar } \\
\text { Sanidad policía }\end{array}$ & $\begin{array}{l}29,4 \% \\
20,7 \% \\
13,1 \% \\
11,3 \% \\
0,92 \% \\
0,39 \% \\
0,36 \% \\
0,21 \% \\
0,13 \% \\
0,13 \% \\
0,10 \% \\
0,10 \% \\
0,07 \% \\
0,05 \% \\
0,02 \%\end{array}$ \\
\hline
\end{tabular}

mujeres $(0,5 \%)$ y por último siete y ocho partos en 5 $(0,2 \%)$ y $2(0,11 \%)$ mujeres respectivamente.

Como puede observarse en la tabla 2, en relación a los Resultados de las citologías, se obtuvieron 8 categorías correspondientes a los diferentes resultados que reportaron las citologías realizados a las mujeres de Armenia en el año 2012, donde el dato que más se repitió fue el de Atipia Celular (ASC-US) con 289 datos correspondientes al 76\% (figura 1).
RESULTADOS CITOLOGIA ARMENIA 2012

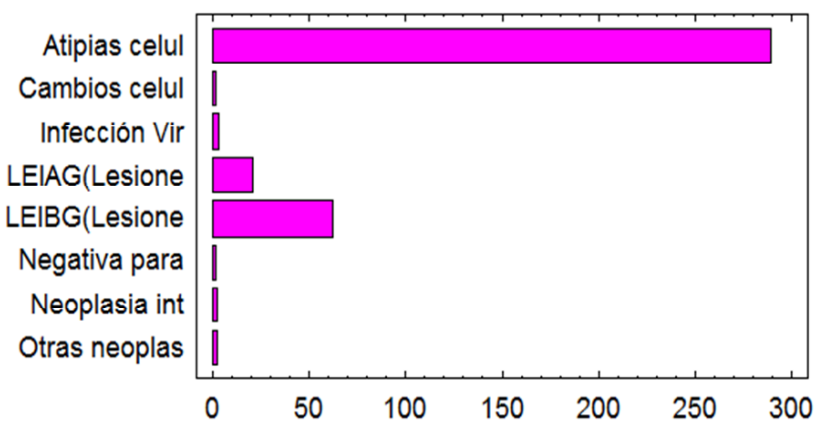

Figura 1. Resultados citología Armenia 2012 
En orden descendente se encuentra LEIBG (lesión intra epitelial de bajo grado) con 62 valores que corresponden a un 16\%, LEIAG (lesión intraepitelial de alto grado) con 20 valores y un $5 \%$ y por último la Infección por el VPH con 3 valores que equivale a un $0,08 \%$. Es decir, 82 citologías, que corresponden a $25 \%$, tuvieron lesión intra epitelial (NIC I, NIC II y NIC III). Por último los resultados que con menor frecuencia fueron reportados por las citologías realizadas fueron, Neoplasia intra epitelial con 2 datos que equivale al $0,05 \%$, Otras neoplasias con 2 valores que corresponden a un $0,05 \%$, Negativa para neoplasia con un solo dato $(0,03 \%)$ y Cambios celulares benignos con un solo dato que corresponde a $0,03 \%$.

El tipo de manejo al cual fueron remitidas las mujeres que se realizaron citologías en Armenia para el año 2012, de acuerdo con número y frecuencia son las siguientes: el tipo de manejo que más se recomendó a las mujeres que se realizaron citología en Armenia para el año 2012 fue biopsia más colposcopia con una frecuencia de 137 que corresponde al 36,05\%, en segundo lugar remisión al ginecólogo con una frecuencia de $69(18,16 \%)$, posteriormente colposcopia con una frecuencia de $60(15,79 \%)$ y por último biopsia endocervical con una frecuencia de $2(0,05 \%)$. Encontramos un notorio subregistro de 112 reportes que corresponden al 29,47\%.

\section{TIPO DE MANEJO}

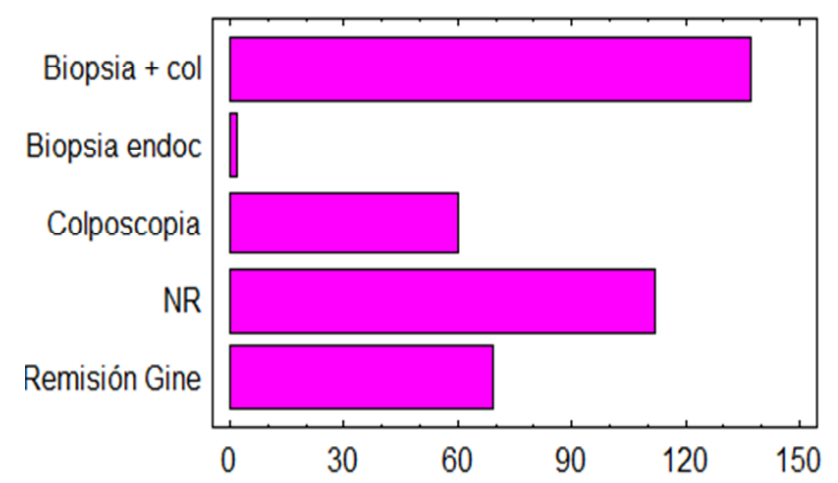

Figura 2. Histograma de Tipo de Manejo

Con relación al método de planificación (figura 3) utilizado encontramos que la mayoría de las mujeres no utilizaba ningún método con una frecuencia de 85 y un porcentaje $22,37 \%$. El siguiente dato que más se repite es el método de planificación definitivo, el quirúrgico, con una frecuencia de $70 \mathrm{y}$ un porcentaje de $18,42 \%$, continuando con el hormonal inyectable presentando una repetición de 34 y un porcentaje de 8,95\%. En los métodos de planificación menos utili- zados encontramos el dispositivo intrauterino con 12 repeticiones y un porcentaje del 3,16\%, luego de este se sitúa el método de barrera con una frecuencia de 8 y con un $1,52 \%$, por último está el implante hormonal con 5 repeticiones correspondiendo al 1,31\%.

\section{MÉTODO DE PLANIFICACIÓN FAMILIAR}

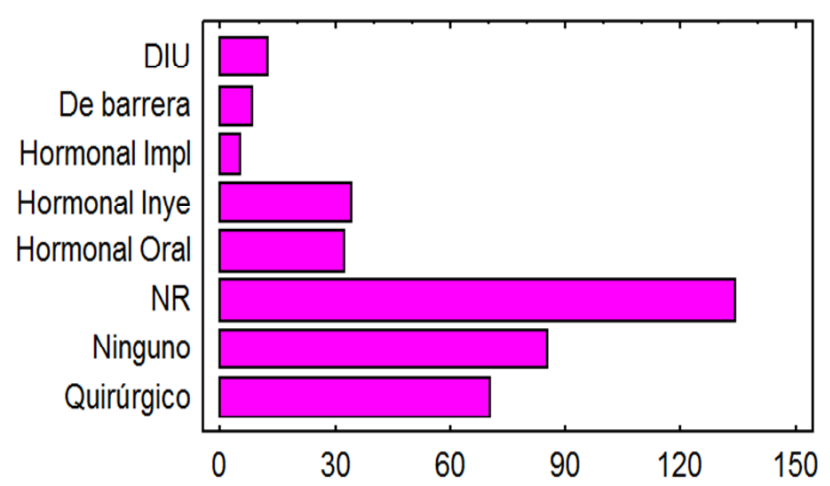

Figura 3. Método de planificación en las mujeres que se realizaron la citología para el año 2012 en Armenia (Quindío).

Es decir, 22,37\% no utilizaba ningún método de planificación, 31,84\% utilizaba métodos como el quirúrgico, hormonal y dispositivo y sólo el 1,52\% de las mujeres que se hicieron la citología utilizaba el condón como medio de planificación y protección.

Según la realización de citologías en las mujeres de la ciudad de Armenia Quindío por EPS e IPS para el año 2012, se presentó que la institución que más realiza citologías es Quindicáncer con una frecuencia de 79 y un porcentaje de 20,79\%, continuando está Sanitas presentando una repetición de 50 y un porcentaje de $13 \%$ y Cosmitet con 43 repeticiones que corresponden a un $11 \%$. Las que realizaron el menor número de citologías fueron Uniquindío con una frecuencia de 4 que corresponden al $0.1 \%$ luego observamos a Caprecom con 3 repeticiones que equivalen al 0,03\%, a continuación está Sanidad Militar con 2 valores que corresponden a un $0,05 \%$ y por último se presenta Sanidad de la policía con 1 repetición que corresponde al $0,02 \%$.

La prueba de chi cuadrado para el manejo recomendado según el resultado de citología fue estadísticamente significativa $(\mathrm{P}=0,0000)$. El resultado de citología atipia celular reporta con mayor frecuencia el manejo biopsia más colposcopia con 98 datos que equivalen a $25,79 \%$, remisión al ginecólogo con 58 datos que equivalen a $15,26 \%$, colposcopia con 43 datos que equivalen a 11,32\% y, biopsia endocervical con 1 dato que equivale a $0,26 \%$. El resultado de citología 
Lesión Intraepitelial de Bajo Grado (LEIBG) reporta con mayor frecuencia el manejo de biopsia más colposcopia con 31 datos que equivalen a $8,16 \%$, seguido de colposcopia con 13 datos que equivalen a 3,42\% y remisión al ginecólogo con 8 datos que equivalen a 2,11\%.El resultado de citología Lesión Intraepitelial de alto Grado (LEIAG) reporta con mayor frecuencia biopsia más colposcopia con 4 datos que equivalen a $1,05 \%$, colposcopia con 3 datos que equivalen a $0,79 \%$ y remisión al ginecólogo 2 datos que equivalen a $0,53 \%$. Es importante resaltar que hay subregistro (NR), en todos los resultados.

La prueba de chi cuadrado para el resultado de citología según el método de planificación familiar fue estadísticamente significativa $(\mathrm{P}=0,0185)$. Se encontró que las mujeres con el resultado de atipias celulares, el dato que más se presentó fue ningún método de planificación, con una frecuencia de 61 que equivale al $16,05 \%$; luego se encuentra el quirúrgico con 58 repeticiones que equivale al $15,26 \%$; continuando con el hormonal oral con 28 repeticiones que corresponde al 7,37\% y entre los métodos de planificación menos usados en cuanto atipia celular encontramos el DIU con 5 repeticiones que corresponde al 1,32\% y por último el implante hormonal que tiene 4 repeticiones equivalentes al 1,05\%. También se encontró subregistro (NR), con una frecuencia de 101 que corresponde al $26,58 \%$

Se encontró que las mujeres con el resultado LEIBG el dato que más se presentó fue ningún método de planificación con una repetición de 13 que equivale al $3,42 \%$, luego se encuentra el quirúrgico con 9 repeticiones que equivale al $2,37 \%$, continuando con el hormonal inyectable con 6 repeticiones que corresponde al 1,58\% y entre los métodos de planificación menos usados en cuanto a LEIBG encontramos el DIU con 3 repeticiones que corresponde al $0,79 \%$ y por último el implante hormonal que tiene repetición que equivalen al $0,26 \%$. El subregistro (NR) presenta una frecuencia de 25 que corresponde al 6,58\%. En cuanto al resultado de LEIAG el dato que más se presentó fue ningún método de planificación con una repetición de 11 que equivale al 2,89\%; luego se encuentra el quirúrgico con 3 repeticiones que equivale al 0,71\%, continuando encontramos el hormonal oral, el hormonal inyectable y el quirúrgico los cuales presentaron 1 repetición que corresponde al $0,26 \%$ y los que no tuvieron ninguna presentación fueron el método de barrera y el implante hormonal. Y el subregistro (NR) con una frecuencia de 3 que corresponde al 0,71\%. También se encontró una relación estadísticamente significativa entre resultados de citología por EPS e IPS, $(\mathrm{P}=0,0000)$, se puede observar que, la EPS Quindicáncer fue la que más citologías reportó, en ésta encontramos 65 resultados de atipia celular $(17,11 \%), 7$ reportes de LEIAG $(1,84 \%), 6$ reportes de LEIBG $(1,58 \%)$ y un reporte Negativo para neoplasia $(0,26 \%)$; en segundo lugar está Sanitas, en la cual encontramos 43 reportes $(11,32 \%)$ con atipia celular, $2(0,53 \%)$ con LEIAG, $4(1,05 \%)$ resultados con LEIBG y un $(0,26 \%)$ resultado con neoplasia intraepitelial; en tercer lugar está Cosmitet con 39 resultados con atipia celular $(10,26 \%), 2$ con LEIAG $(0,53 \%)$, uno con LEIBG $(0,26 \%)$ y un resultado de Neoplasia intraepitelial $(0,26 \%)$; en cuarto lugar, Coomeva reporta 22 resultados de atipia celular $(5,79 \%)$, un resultado de Infección por VPH $(0,26 \%)$ y 12 resultados de LEIBG (3,16\%); Saludcoop en quinto lugar, reportó $10(2,63 \%)$ resultados con atipia celular, uno $(0,26 \%)$ con LEIAG, $3(0,79 \%)$ con LEIBG y un $(0,26 \%)$ resultado para la categoría otras neoplasias; Comfenalco Antioquia con 10 reportes de atipia celular $(2,63 \%)$ y 4 reportes con LEIBG $(1,05 \%)$; Cafesalud reportó 7 resultados de atipias celulares que corresponde al $1,84 \%$ y un solo reporte de LEIBG $(0,26 \%)$.

En los últimos lugares encontramos a RedSalud con 2 reportes $(0,53 \%)$ para atipia celular y LEIBG en cada una respectivamente; Salud Total reportó 5 (1,32\%) resultados con atipia celular; Comfenalco Quindío con un reporte con atipia celular $(0,26 \%), 2$ reportes con LEIAG $(0,53 \%)$ y uno con LEIBG $(0,26 \%)$; el centro de salud de la Universidad del Quindío con 4 $(1,05 \%)$ resultados de atipia celular; Caprecom reportó 2 resultados de atipia celular $(0,53 \%)$ y un reporte de LEIBG (0,26\%);Sanidad Militar reportó un $(0,26 \%)$ resultado con cambios celulares y $1(0,26 \%)$ con LEIBG; y por último en la EPS Sanidad Policía encontramos solo un reporte $(0,26 \%)$ para atipia celular.

\section{DISCUSIÓN}

Con respecto a la edad el promedio encontrado fue de 37,4 años y el rango está entre 15 y 79 años. Esto es clara evidencia de que hay mujeres que se están realizando la citología por debajo de los 25 años y por encima de los 69 años, que se recomienda en los protocolos. Podemos también decir que aquí se evidencia 
la importancia de que la citología se empiece a más temprana edad y hasta más tarde, que lo sugerido por el protocolo de la Liga contra el Cáncer y del Ministerio de Salud (3).

En cuanto al número de gestaciones el rango se encontró entre los mismos intervalos de la paridad, en primer lugar se encuentra tres gestaciones $(30,5 \%)$, en segundo lugar dos gestaciones $(25,2 \%)$ pacientes, le sigue una única gestación (16.47\%), cuatro gestaciones $(15.88 \%)$, continua cinco gestaciones $(0,72 \%)$ y por ultimo siete $(0,29)$ y ocho $(0,11 \%)$ gestaciones. Lo mostrado en el estudio sobre los factores de riesgo gineco obstétricos para el cáncer cérvico uterino en la atención primaria de salud (4), se encontró un predominio de casi todos los tipos de carcinoma en las mujeres que habían parido 1 o 2 veces $(44,0$ y $36,0 \%$, respectivamente). $\mathrm{Y}$ un $16,0 \%$ de las que tuvieron 3 o más partos.

Respecto al número de gestaciones, la bibliografía nos dice que los cambios inducidos por el embarazo como el estado de inmunosupresión podría aumentar la susceptibilidad del organismo a los agentes infecciosos en este caso el VPH e influir en el riesgo de progresión de esta patología (5), teniendo en cuenta ésto algunos estudios reportan que a mayor número de gestaciones mayor riesgo de carcinoma invasivo/ in situ. En el estudio Multicenter Cervical Center Study Group de la International Agency for Research on Cancer (IARC), el riesgo de carcinoma en mujeres con 7 embarazos era 4 veces mayor que en la mujer nulípara y el riesgo aumentaba con el número de embarazos (6).

Si bien la gestación y el parto conforman un hecho fisiológico en la vida reproductiva de la mujer, la bibliografía muestra que el cáncer cérvicouterino es más común en las mujeres que ya tienen hijos que en las nulíparas, esto se asocia al hecho de que las mujeres con mayor número de embarazos inician tempranamente su vida sexual y su primer contacto. Las lesiones e infecciones del cuello uterino, que suceden durante el parto, alteran los límites normales entre los 2 epitelios y el número de embarazos llegados a término, debido a algún grado de inmunosupresión que tiene la gestante, puede ser un componente favorecedor del cáncer.

En el presente estudio, el resultado que presentó una mayor frecuencia fue el de atipia celular con $76 \%$, mientras que en estudio sobre la prevalencia de anormalidades citológicas e histológicas de cuello uterino en un grupo de mujeres en Bogotá, en el año 2010 se encontró que fueron informados un $24,7 \%$ con atipia celular (7).

Con respecto a la lesión intraepitelial de bajo grado y la lesión intraepitelial de alto grado en nuestro estudio encontramos que se reportó en un 16\% y 5\% respectivamente, en el estudio realizado en Bogotá se reportó LEIBG en un 4,32\% y LEIAG en un 1,21\%. En cuanto a la infección por VPH se encontró en una menor proporción $(0,08 \%)$ en relación a un $6,2 \%$ en el estudio realizado en la ciudad de Bogotá. Y la neoplasia intraepitelial que en este estudio se presentó en un $0,03 \%$, en el estudio de Bogotá fue de 7,54\%.

Es posible afirmar que en el estudio en Armenia en el año 2012, la citología está detectando más tempranamente las atipias celulares que en el estudio de Bogotá en el año 2010.

Observamos que el manejo de los hallazgos anormales más recomendado a las mujeres que se realizaron citología en Armenia para el año 2012 fue biopsia más colposcopia con $36,05 \%$, seguido de remisión al ginecólogo (18,16\%), posteriormente colposcopia $(15,79 \%)$ y por último biopsia endocervical $(0,05 \%)$. Al comparar el manejo respecto a los marcos normativos vigentes (8), es posible afirmar que en el Municipio de Armenia se remite tempranamente a colposcopia + biopsia y al ginecólogo, frente a la presencia de alguna anormalidad.

Según la literatura, de acuerdo con los resultados de la citología las conductas a seguir son:

- Si el reporte de resultados es normal satisfactoria, se continua el esquema de tamizaje 1-1-3

- Si reporta cambios benignos, se analizan las causas, se ordena el tratamiento necesario y continuo el esquema de tamizaje.

- Si reporta como anormal, la paciente debe ser remitida a una unidad de patología cervical y colposcopia (8).

Sin embargo, a pesar que las atipias encontradas fueron de $76 \%$, sólo un $15,79 \%$ fueron remitidas de colposcopia y el 36,05\% se remitieron a colposcopia+biopsia.

En Armenia se encontró que el método de planificación más usado por las mujeres que se realizaron la citología en el año 2012, fue ninguno (22,37\%), se- 
guido del método quirúrgico $(18,42 \%)$, el hormonal inyectable con $8,95 \%$ y el hormonal oral con $8.42 \%$; los condones y los métodos de barrera son los menos utilizados $(1,52 \%)$.

Según la panorámica que muestra profamilia en el documento de conocimiento y uso de planificación familiar del año 2011 la esterilización femenina es el método más usado con cerca del $39 \%$, continuando con $21,7 \%$ que corresponde a las mujeres que no están usando métodos anticonceptivos, luego está el inyectable con $11,7 \%$ seguido por el hormonal oral con $6,1 \%$. Entre los menos utilizados se encuentra el dispositivo intrauterino con 5,8\%, el condón con 5,2\% y el implante hormonal con 4,3\% (9).

Es decir, el presente estudio encontró un porcentaje similar al de profamilia el no uso de métodos de planificación 22,37 y $21 \%$ respectivamente, al igual que en el uso de métodos hormonales $17,37 \%$ para este estudio y $17,8 \%$ para el de profamilia. Y lastimosamente el método de barrera (condón) para protección está en ambos estudios en penúltimo lugar, con $1,52 \%$ en este estudio y con 5,2\% para el estudio de profamilia. Sin embargo, en este estudio en primer lugar se encontró que las mujeres no están planificando y segundo lugar están utilizando el método quirúrgico. Los demás métodos de planificación se encuentran en el mismo orden de descendencia y similar porcentaje de uso.

Lo anterior es preocupante en materia de promoción y prevención, pues el hecho de que haya un gran porcentaje de mujeres que no están usando métodos de planificación (21,7\%), sumado al método quirúrgico y el uso de anticonceptivos, pone en evidencia que sólo el 1,58\% de las mujeres que se hicieron la citología previene la trasmisión del VIH y otras enfermedades de trasmisión sexual por medio del condón $(10,11)$. Además de lo ya mencionado cabe comentar que las píldoras anticonceptivas son un factor de riesgo para el cáncer de cuello uterino, que se duplicó en las mujeres que tomaron píldoras anticonceptivas por más de 5 años, pero el riesgo regresó a lo normal después de 10 años de haber dejado de tomarlas (12).

Respecto al manejo según diagnóstico se encontró como primer diagnóstico atipia celular a la cual su manejo con mayor frecuencia fue biopsia más colposcopia en un $25,79 \%$, respecto a la bibliografía se evidencia que el manejo indicado es el seguimiento con citología (13), pero es importante aclarar que en este estudio no existía información para saber si estas son citologías de seguimiento o primera vez. Como segundo ítem de manejo para la atipia celular encontramos un $23 \%$ con no registra lo cual se puede entender como un subregistro o que se pierde el seguimiento a las mujeres.

Para las lesiones Intraepiteliales de Bajo Grado el manejo según la bibliografía es la biopsia más colposcopia (13), en este estudio se encontró que el 16\% de las mujeres tenía este diagnóstico de las cuales el $50 \%$ recibieron el manejo según la bibliografía. Para las Lesiones Intraepiteliales el manejo según la bibliografía es procedimiento de escisión electroquirúrgica y observación con esquema de seguimiento con colposcopia (13).

En el presente estudio se encontró que hay 15 EPS e IPS, que prestaron el servicio para la realización de las citologías, la que más fue Quindicáncer con un porcentaje de $20,79 \%$, continuando Sanitas (13\%) y Cosmitet (11\%). En la bibliografía no se encuentran datos sobre el número de citologías que realizan las EPS e IPS en las mujeres de Armenia (Quindío), ó en otras partes de Colombia.

\section{CONCLUSIONES}

Se concluye que la población de mujeres que se realizaron la citología cérvico uterina durante el año 2012 en la ciudad de Armenia, tenían edades entre los 15 y 79 años, es decir que la realización de las citologías se está iniciando a edades más tempranas y se continúan a edades mayores de las recomendadas por el protocolo de la Liga contra el cáncer y el Ministerio de Salud, de allí la importancia de hacer la citología a menor y mayor edad; así mismo la gestación promedio de estas mujeres que se realizaron la citología fue de $1,86 \%$ y la paridad fue de $1,74 \%$. $34 \%$ y $30 \%$ de estas mujeres tuvieron 3 gestaciones y 3 partos. Respecto a los resultados de anormalidad que se reportaron en las citologías realizadas corresponden a atipia celular (ASC-US) en un 76\%, seguido por LEIBG (NIC I) (16\%), LEIAG (NIC II o III) (5\%), infección por VPH $(0,08 \%)$, Neoplasia intraepitelial $(0,05 \%)$, Otras neoplasias $(0,05 \%)$, Negativa para neoplasia $(0,03 \%)$ y Cambios celulares benignos $(0,03 \%)$. Además encontramos que el tipo de manejo que más se recomendó fue biopsia más colposcopia en un $36,05 \%$, en segundo lugar remisión al ginecólogo (18,16\%), posteriormente colposcopia $(15,79 \%)$ y por último 
biopsia endocervical $(0,05 \%)$. Se estableció que el mayor número de citologías realizadas corresponde a la IPS Quindicáncer con un porcentaje de 20,79\%, continuando Sanitas (13\%) y Cosmitet (11\%).

Sobre los métodos de planificación familiar, el 22,37\% no utilizaba ningún método de planificación, 31,84\% utilizaba métodos como el quirúrgico, hormonal y dispositivo y sólo el 1,52\% de las mujeres que se hicieron la citología utilizaba el condón como medio de planificación y protección.

Se evidenció a través del análisis estadístico que la gran mayoría de las mujeres que se realizaron la citología presentaron un gran porcentaje de atipia celular $76 \%$ y que para esta anormalidad el manejo indicado con mayor frecuencia fue biopsia más colposcopia $(36,05 \%)$, seguido de remisión al ginecólogo $(18,16 \%)$, colposcopia $(15,79 \%)$, y biopsia endocer$\operatorname{vical}(0,05 \%)$.
En cuanto a los métodos de planificación según los resultados anormales en el presente estudio se encontró que las atipias celulares están más relacionados con las mujeres que no estaban usando algún método de planificación y que éste presentó un porcentaje cerca del $16,05 \%$, seguido por el quirúrgico $(15,26 \%)$, continuando con el hormonal oral $(7,37 \%)$.

Estos resultados evidencian que es necesario desarrollar mayores esfuerzos y en programas de prevención de neoplasia cervical en la región por medio del fomento del uso del condón como método de protección y planificación.

\section{AGRADECIMIENTOS}

A la Secretaría de Salud del municipio de Armenia. A la enfermera Carmelita Monroy Jaramillo, a la epidemióloga Doctora Liliana Álvarez Quintero y, a la Ingeniera de Sistemas Romelia González Quintero.

\section{BIBLIOGRAFÍA}

1. Liga Colombiana contra el cáncer. Cáncer cérvicouterino. 2008

2. Secretaria de Salud de Armenia Quindío. Pirámide Poblacional de Armenia. Plan Decenal de Salud. 2013.

3. Ministerio de salud. Dirección general de promoción y prevención. Norma técnica para la detección temprana del cáncer de cuello uterino y guía de atención de lesiones preneoplásicas de cuello uterino. 2002.

4. Dunán L, Cala L, Infante N, Hernández T. Factores de riesgo gineco obstétricos para el cáncer cervicouterino en la atención primaria de salud. Revista médica cubana. 2010.

5. Castañeda M, Toledo R, Aguilera M. Factores de riesgo para cáncer cérvicouterino en mujeres de Zacatecas. Revista Salud Pública Méx 1998; Vol. 40(4):330-38

6. Arenas R, Henríquez D, González M. Cáncer de cuello uterino en mujeres menores de 35 años y mayores de 60 años. Revista de obstetricia y ginecología Venez. 2011; 71(4): 252-64.

7. González M, Murillo R, Osorio E, Gamboa O, Ardila J. Prevalencia de anormalidades citológicas e histológicas de cuello uterino en un grupo de mujeres en Bogotá, Colombia. Revista Colombiana de Cancerología 2010; 14(1):22-8.

8. Ministerio de salud y protección social. Resolución 412 del 2000. Norma técnica para la detección temprana del cáncer de cuello uterino y guía de atención de lesiones preneoplásicas de cuello uterino. Colombia.

9. Profamilia. Planificación Familiar: conocimiento y uso. Encuesta en Zonas Marginadas, 2011.

10. The American College of obstetricians and gynecologists. Women's health care physicians. El cáncer de cuello uterino. Patienteducation. 2013.

11. Liga contra el cáncer. Seccional Bogotá. Cáncer cérvico uterino. 2013

12. Asociación Americana de Cáncer. Informe Cáncer de cuello uterino. 2013.

13. Instituto nacional de cancerología. Colombia. Guía de atención integral para la tamización, detección temprana, diagnóstico, tratamiento y seguimiento de pacientes con lesiones pre invasivas del cuello uterino; diagnóstico, tratamiento y seguimiento de pacientes con cáncer de cuello uterino. Documento alcance y objetivos de la guía de práctica clínica. Noviembre de 2012. 